\title{
MONTE CARLO STUDY OF SELECTIVE MAGNETIC DILUTION IN SPINEL STRUCTURE
}

\author{
L. PYTLIK \\ Faculty of Physics and Nuclear Techniques, Academy of Mining and Metallurgy \\ Al. Mickiewicza 30, 30-059 Kraków, Poland
}

In the present work results of a Monte Carlo study of magnetic ordering and percolation tresholds in selectively diluted spinel structure are discussed. Classical spin model with the Heisenberg type interactions was used with additional cubic anisotropy terms. Three types of selective dilution were studied: separate dilutions of $A$ and $B$ sublattices and a homogeneous dilution of both sublattices. The results are compared for various degrees of magnetic competition between first and second neighbours interactions.

PACS numbers: $75.10 . \mathrm{Hk}, 75.10 . \mathrm{Nr}, 75.40 . \mathrm{Mg}, 75.50 . \mathrm{Lk}, 75.50 . \mathrm{Gg}$

\section{Introduction}

The connections between the percolation phenomena and magnetic ordering has been studied for a long time and the basic concepts are more or less clear. When some of the magnetic ions are replaced by non-magnetic ions it is usually described as magnetic site dilution. For a given lattice topology there is a critical concentration $X_{\mathrm{C}}$ of magnetic ions which is required for formation of magnetic order in low temperatures. Description of various aspects of magnetic order and spin glass phases appearing near the percolation treshold can be found in $[1,2]$. The percolation phenomena are more complicated for compounds in which more than one magnetic sublattice is present. It turns out that the effect of non-magnetic ions depends on their location in the structure. In order to describe this the concept of selective magnetic dilution has been introduced [3].

An ideal system for experimental studies of the selective dilution phenomenon is provided by the spinel structure, where two non-equivalent magnetic sublattices (A and $B$ ) are present. General formula for a magnetically diluted spinel-type compound reads: $\left[\mathrm{A}_{X_{\mathrm{A}}}, \mathrm{N}_{1-X_{\mathrm{A}}}\right]\left[\mathrm{B}_{X_{\mathrm{B}}}, \mathrm{N}_{1-X_{\mathrm{B}}}\right]_{2} \mathrm{O}_{4}$, where $X_{\mathrm{A}}$ and $X_{\mathrm{B}}$ denote the concentrations of magnetic ions in the $A$ and $B$ sublattices respectively. Spinels provide a good example for short interaction range system, where the antiferromagnetic nearest neighbour (nn) A-B interaction is the dominant one, and the second neighbours interactions ( $\mathrm{A}-\mathrm{A}$ and $\mathrm{B}-\mathrm{B}$ ) are for most cases in conflict with $A-B$. In the last two decades many examples of spin-glass phases has been found 
in the diluted spinel systems [4-7]. The first paper on selective dilution [3] considered the spinel system with nearest neighbours interactions only. For such a system the $\mathrm{nn}$ percolation tresholds have been calculated for selective dilutions of $\mathrm{A}$ and $B$ sublattices and homogeneous dilution of the whole system. Unfortunately, the neglect of $\mathrm{A}-\mathrm{A}$ and $\mathrm{B}-\mathrm{B}$ interactions resulted in considerable differences between the calculated and experimental results. Other propositions for the actual shape of the diagram, with A-A and B-B interactions included, can be found in [8-10]. Another shape of the diagram has been suggested in a numerical study using the magnetic energy minimization [11]. In the present paper the percolation phenomena observed during selective magnetic dilution of the system have been studied numerically using the Monte Carlo methods.

\section{The model and simulation characteristics}

The lattice spins were represented by classical vectors of unit length $\sigma$ with orientation described by the $\theta$ and $\phi$ angles of the spherical coordinate system. The local spin energies required for the Metropolis algorithm has been expressed as dot products of the local spin vector and the local effective field, calculated as a vector sum of the external field and the sum of contributions from the nearest and next nearest neighbours. The final formulas can be expressed using the following interaction parameters:

$$
j_{\mathrm{AB}}=J_{\mathrm{AB}} S_{\mathrm{A}} S_{\mathrm{B}}, \quad j_{\mathrm{A}}=\frac{-J_{\mathrm{AA}} S_{\mathrm{A}}}{J_{\mathrm{AB}} S_{\mathrm{B}}}, \quad j_{\mathrm{B}}=\frac{-J_{\mathrm{BB}} S_{\mathrm{B}}}{J_{\mathrm{AB}} S_{\mathrm{A}}} .
$$

The model system contained between $15 \times 15 \times 15$ and $30 \times 30 \times 30$ unit cells. Typical run consisted of a field cooling and then a zero field heating. During the calculation various parameters were extracted including: average sublattice magnetization, total magnetic energy, Fourier transforms for both sublattices, local bond angles etc. In the postprocessing stage the heat capacity as a function of temperature and correlation functions for selected system dumps were calculated. In the final stage the magnetic ordering temperatures and magnetic parameters of the system have been plotted as functions of $X_{\mathrm{A}}$ and $X_{\mathrm{B}}$, the concentrations of magnetic ions in $\mathrm{A}$ and $B$ sublattices respectively. Below the results for three special cases of magnetic dilution are presented for $j=j_{\mathrm{A}}=j_{\mathrm{B}}$ equal to $0.05,0.25$, and 0.5 with $j_{\mathrm{AB}}$ value taken as $200 \mathrm{~K}$.

\section{Homogeneous dilution}

The $X_{\mathrm{A}}=X_{\mathrm{B}}$ cut of the $\left(X_{\mathrm{A}} n, X_{\mathrm{B}}\right)$ phase diagram leads from a fully ordered ferrimagnetic phase in low temperatures for $X_{\mathrm{A}}=X_{\mathrm{B}}=1$ to a paramagnetic phase for $X_{\mathrm{A}}=X_{\mathrm{B}}=0$. The nearest neighbours percolation treshold calculated in [3] is $X_{\mathrm{C}}=0.227$. The neglection of $\mathrm{A}-\mathrm{A}$ and $\mathrm{B}-\mathrm{B}$ interactions is equivalent to $j=0$ case.

In Fig. 1a the A-A and B-B spin-spin correlation functions values for $r=5 a$ (about $50 \AA$ ) are compared for $j=0.05$ and $j=0.5$ cases. The difference between the two cases is clearly visible but there are also similarities. Both curves for $T=10 \mathrm{~K}$ exhibit a change in slope while the $T=30 \mathrm{~K}$ curves go more or less directly to zero at a given concentration. The plotted $T_{\mathrm{C}}-X_{\mathrm{A}}$ diagrams showed 

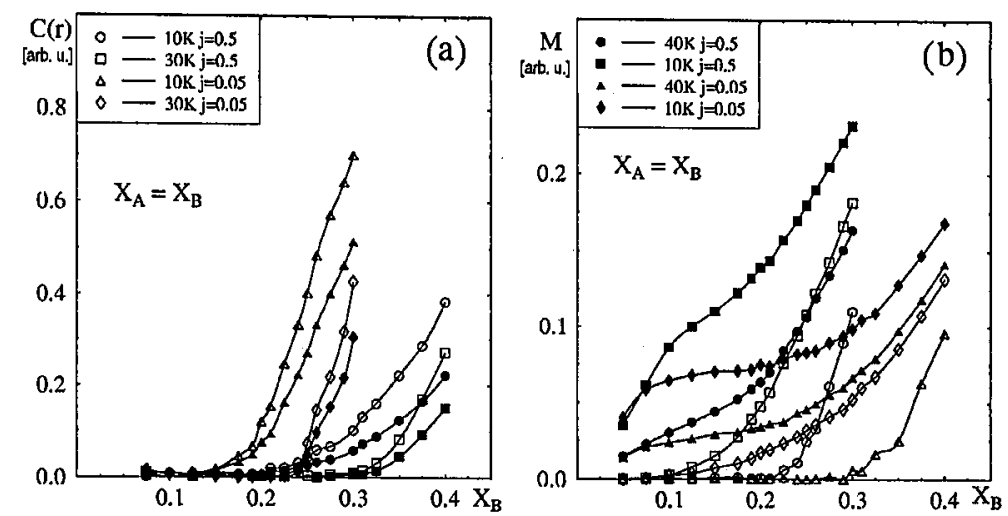

Fig. 1. (a) Spin-spin correlation functions A-A (open symbols) and B-B (full symbols) for $X_{\mathrm{A}}=X_{\mathrm{B}}$ as a function of $X_{\mathrm{A}}$. (b) In field and remanent magnetization values for the $j=0.05$ and $j=0.05$ cases.

that these concentrations are close to values obtained from $T_{\mathrm{C}}\left(X_{\mathrm{A}}\right)$ extrapolation to zero. The short range correlation region visible for $T=10 \mathrm{~K}$ i.e. $X_{\mathrm{A}}<0.2$ for $j=0.05$ and $X_{\mathrm{A}}<0.27$ for $j=0.5$ case indicate a frozen, spin-glass type phase. This is also confirmed by the remanent magnetization values (see Fig. 1b) which is nonzero down to $X_{\mathrm{A}}=0.12$ for both cases. Also an inflection point is clearly visible at the field curves. It seems that the 0.12 value is close to percolation treshold calculated for next nearest neighbours. The percolation treshold calculated in [3] seems to coincide with the critical concentration obtained from the extrapolation of the ferri ordering temperature down to zero for $j=0.05$ case.

\section{Selective dilution of $\mathbf{A}$ and $\mathbf{B}$ sublattice}

The cases when only one sublattice is being diluted are essentially different. The A sublattice dilution leads from the ferrimagnetic phase for $X_{\mathrm{A}}=X_{\mathrm{B}}=1$ to a very strange, highly frustrated non-collinear phase with short range magnetic order for $X_{\mathrm{A}}=0, X_{\mathrm{B}}=1$ [5]. The obtained results for correlation functions, magnetization sublattices and $T_{\mathrm{C}}\left(X_{\mathrm{A}}\right)$ diagram show that there is a critical concentration for existence of non-collinear ferri phase, which is strongly dependent on the $j_{\mathrm{B}}$ value. The critical concentration for $j_{\mathrm{B}}=0.05$ case is close to 0.09 calculated in [3] while the value for $j_{\mathrm{B}}=0.5$ is higher than 0.2 . Below that critical value a frozen cluster phase is visible (from the correlation functions), and the clusters are formed by $\mathrm{A}$ type ions polarizing the softly ordered B sublattice neighbourhood.

On the other hand, the $B$ sublattice dilution ends up in a proper antiferro order on the A sublattice for $X_{\mathrm{A}}=1, X_{\mathrm{B}}=0$. For this case the exact behaviour qualitatively depends on the $j$ value. For $j=0.5$ case there is a mixed phase region, where both types of order are visible, i.e. antiferro for A sublattice (perpendicular components) and ferri on both sublattices (longitudinal components). The order parameters (respective Fourier peaks) are presented in Fig. 2b. It can be seen that the mixed phase region ranges from $X_{\mathrm{B}}=0.07$ to $X_{\mathrm{B}}=0.22$ for $T=10 \mathrm{~K}$ and it 

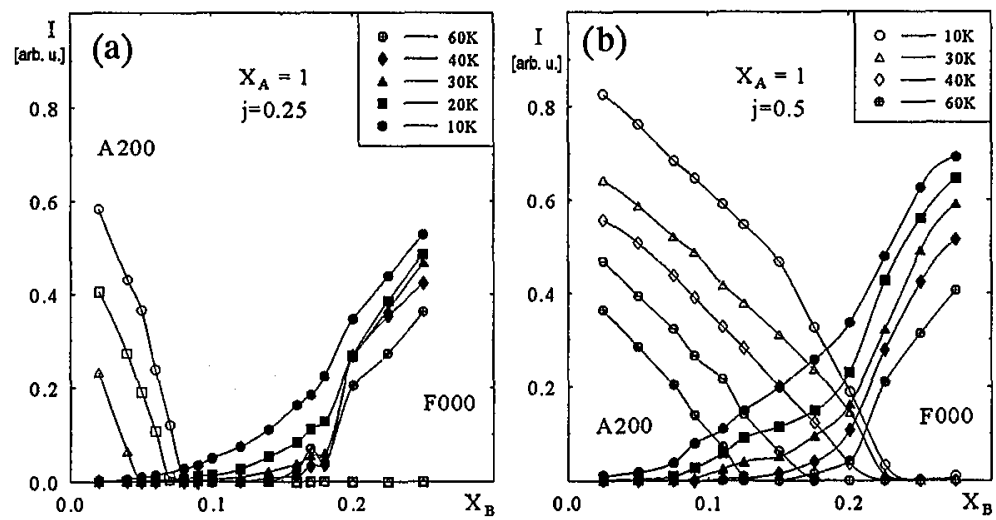

Fig. 2. The antiferro and ferri order parameters for $X_{\mathrm{A}}=1$ exhibited by respective Fourier peaks for the $j=0.25$ (a) and $j=0.5$ (b) cases.

ceases above $T=40 \mathrm{~K}$. On the other hand, the $j=0.25$ case (Fig. $2 \mathrm{a}$ ) shows that the ferri phase extrapolates down to zero near $X_{\mathrm{B}}=0.17$ while the antiferro phase reaches at most $X_{\mathrm{B}}=0.07$ for $T=10 \mathrm{~K}$. It is evident that there is a short range order phase in between. The obtained results are similar to some cases presented in $[7]$.

\section{Conclusions}

From the selected results presented in the paper it can be seen that such a Monte Carlo study can be an efficient tool for studying percolation phenomena in diluted and substituted compounds. However for detailed quantitative comparison of the percolation treshold the analysis of size scaling effects would be required.

\section{References}

[1] D. Stauffer, Phys. Rep. 54, 1 (1979).

[2] K. Binder, P. Young, Rev. Mod. Phys. 58, 801 (1986).

[3] F. Scholl, K. Binder, Z. Phys. B 39, 239 (1980).

[4] D. Fiorani, J. Appl. Phys. 69, 4774 (1991).

[5] R.A. Brand, H. Georges-Gilbert, J. Hubsch, J.A. Heller, J. Appl. Phys. 69, 4774 (1991).

[6] J.L. Soubeyroux, J. Phys. (France), Coll. C8 49, 1117 (1988).

[7] J.L. Dormann, M. Nogues, J. Phys. C, Condens. Matter 2, 123 (1990).

[8] J. Hubsch, G. Gavoille, J. Bolfa, J. Appl. Phys. 49, 1363 (1978).

[9] J. Villain, Z. Phys. B 33, 31 (1979).

[10] C.P. Poole, H.A. Farach, Z. Phys. B 47, 55 (1982).

[11] L. Pytlik, J. Phys. IV (France) 7, C1-183 (1997). 